\title{
Composición química proximal y morfométría de cholga (Aulacomya ater, Molina, 1782) y chorito (Mytilus chilensis, Hupé, 1854) comercializados en la Región de Magallanes
}

\author{
Proximal chemical composition and morphometry of the ribbed \\ (Aulacomya ater, Molina 1782) and the blue (Mytilus chilensis, Hupé, 1854) \\ mussels commercialized in the Region of Magallanes
}

Viviana Ríos $^{1}$, Ninoska Ocampo ${ }^{1} \& \mathrm{M}^{\mathrm{a}}$. S. Astorga-España ${ }^{2}$

\section{Resumen}

Se estudió la composición química proximal, los parámetros morfométricos y la cantidad de parte comestible en cholga (A. ater) y chorito ( $M$. chilensis) recolectados en estaciones del estrecho de Magallanes, de lugares adyacentes a parques Nacionales. En chorito, las muestras provenientes de bahía Gente Grande, que es el único sector de muestreo, cercano a la costa de la Isla de Tierra del Fuego, en el lado oriental del estrecho de Magallanes, se determinaron los parámetros morfométricos mayores. Mientras que la mayoría de los parámetros morfométricos mayores determinados en cholga son de las muestras provenientes de puerto Edén, en el lado occidental del estrecho de Magallanes. La carne de fresca de chorito y cholga presentó un alto contenido de humedad. Las proteínas son el componente bioquímico con mayor porcentaje, seguido por los hidratos de carbono y en menor cantidad las grasas, en ambos moluscos estudiados. Importante destacar también, que las cholgas presentan mayor cantidad de carbohidratos y energía (Kcal/100 g) que los choritos. Las diferencias observadas entre los choritos y las cholgas, en los parámetros morfométricos, la cantidad de carne fresca y la composición química, se atribuyen a las características propias de cada especie, las cuales al ser comparadas en este estudio con tablas de composición de alimentos nacionales e internacionales muestran una buena calidad de los organismos para el consumo humano.

\section{Palabras Clave:}

Bivalvos, nutricional humana, composición proteica y carbohidratos,

especies de interés comercial

\section{Abstract}

We examined the proximal chemical composition, morphometric parameters and the amount of edible portion in the Chilean ribbed mussel (A. ater) and blue mussel ( $M$. chilensis) collected from sites along the Magellan Strait, mainly near National parks at the Sub-Antarctic Magellan region, Chile. The higher morphometric parameters were obtained from blue mussels sampled in bahía Gente Grande, which is the only site near the coast of Tierra del Fuego island, on the Eastern side of the Strait of Magellan. The higher biometric parameters were obtained from ribbed mussels sampled in Puerto Edén, on the western side of the Strait of Magellan. The fresh meat of both mussels had high moisture content. The highest percentage of biochemical component in both bivalve species was protein, followed by carbohydrates and, to a lesser extent, lipids. It is important to note that the ribbed mussel has a greater amount of carbohydrates and energy (Kcal/100 g) than the blue mussels. The differences observed between both specie

\footnotetext{
1 Programa de Magíster en Ciencias Mención Manejo y Conservación de Recursos Naturales de Ambientes Subantárticos. Universidad de Magallanes, P.O. Box 113-D, Punta Arenas, Chile.

2 Departamento de Ciencias y Recursos Naturales, Avenida Bulnes \#01855, Universidad de Magallanes, P.O. Box 113-D. Punta Arenas, Chile;

$\varangle$ msoledad.astorga@umag.cl
} 
in biochemical compositions, morphometric parameters and in the amount of fresh meat, may be explained by the biological characteristics of each species. A comparison of our results to parameters in international and national tables of food composition, revealsthat the commercial bivalve organisms examined in this study are of very good nutritional quality.

\section{Key Words:}

Bivalves, human nutrition, protein and carbohydrate composition, species of commercial interest.

\section{INTRODUCCIÓN}

Los moluscos bivalvos, frescos y procesados, desempeñan un papel cada vez más importante en la dieta para la población humana a nivel mundial (Farías, 2008). En la actualidad existe una mayor preocupación por conocer la calidad del alimento que se está ingiriendo tanto del punto de vista nutritivo, como organoléptico e higiénico-sanitario. Muchos trabajos se han desarrollado acerca de la composición química proximal de la carne y la calidad nutricional del Mytilus galloprovincialis de diferentes orígenes (Orban et al. 2002; Freites et al. 2002, 2003; Fuentes et al. 2009).

Los pescados y los mariscos son una importante fuente de alimento para los seres humanos por la calidad y cantidad de nutrientes que aporta. Estos, se sitúan en la mayor parte del mundo como alimentos proteicos básicos, después de la carne de mamíferos y aves y, en ciertos países como Japón y Corea son la principal fuente proteica. Una ración promedio de $100 \mathrm{~g}$ de estos alimentos, cubre más del 50\% de la ingesta diaria de proteínas recomendada por la FAO, las cuales son proteínas de elevado valor biológico y que presentan una digestibilidad superior al 80\% (FAO/WHO, 1988).

Por lo tanto los moluscos son una fuente de nutrientes, y el consumo de estos productos proporciona una fuente barata de proteínas de alto valor biológico para el consumo humano. Además, contribuyen a la ingesta de minerales esenciales y vitaminas (Astorga-España et al. 2007).

Los moluscos comestibles más importantes son los bivalvos y gastrópodos los cuales se pueden comercializar al igual que los pescados frescos, precocidos, en conservas y marinados (Cabello et al. 2004). En Chile, los moluscos bivalvos como cholgas y choritos, son de gran importancia comercial y su forma de consumo es como producto fresco, congelado, semideshidratado y ahumado (Carranza et al. 2009).

Las cholgas (Aulacomya ater) son organismos bentónicos costeros de aguas someras que viven formando grandes bancos adheridos a sustratos duros y rocosos a través de su biso alcanzando profundidades de hasta 70 metros (Zúñiga, 2002), alimentándose principalmente de fitoplancton y detritos orgánicos. A. ater se distribuye por la costa del Pacífico desde Callao (Perú) hasta el Canal Beagle, isla Navarino e isla Picton (Chile), continuando por la costa Atlántica hacia el norte hasta el sur de Brasil. También se puede encontrar en la isla Juan Fernández (océano Pacífico) y las Malvinas (océano Atlántico) (Uriarte, 2008).

El chorito (Mytilus chilensis) es una especie endémica de Chile se distribuye desde Arica al Cabo de Hornos, siguiendo por el Atlántico hasta las islas Malvinas. Ubicándose desde la zona del intermareal hasta los $25 \mathrm{~m}$ de profundidad, puede habitar lugares con alta salinidad y ambientes salobres como desembocaduras de ríos o cercanía de glaciares, alimentándose principalmente de microalgas por filtración (Osorio, 2002).

En la zona de Magallanes es posible contar con importante información sobre la diversidad y heterogeneidad en tipos de hábitat marino costeros de la biota marina, principalmente de descripción de moluscos, macrofauna asociada, taxonomía, ecología, distribución y biogeografía de diversas especies, en diferentes sectores del Estrecho de Magallanes (Langley et al.1980; Aldea \& Rosenfeld, 2011; Andrade \& Brey, 2014). Sin embargo, existe escasa información sobre los parámetros morfométricos y la composición química en choritos y cholgas que habitan en bancos naturales del estrecho de Magallanes.

El objetivo de este trabajo fue determinar los parámetros morfométricos, la cantidad de carne comestible y la composición química proximal de cholgas (Aulacomya ater) y choritos (Mytilus chilensis), provenientes de bancos naturales infralitorales, de sectores estratégicos del Estrecho de Magallanes, y que son consumidos por la población de Magallanes. 


\section{MATERIAL Y MÉTODOS}

\section{Área de estudio y muestreo}

El área de estudio corresponde a estaciones de muestreo ubicadas en el Estrecho de Magallanes, un complejo canal natural de conexión entre los océanos Pacífico y Atlántico, localizado en el extremo sur de Sudamérica, entre la Patagonia Chilena, y la Isla Grande de Tierra del Fuego (Panella et al. 1991; Antezana, 1999). Entre las características batimétricas más notables, el Estrecho de Magallanes puede ser dividido en tres micro-cuencas: la oriental, ubicada entre la entrada del Océano Atlántico y la Segunda Angostura, la central desde Segunda Angostura hasta la Isla Carlos III, y la occidental desde la Isla Carlos III hasta la entrada del Océano Pacífico (Valdenegro \& Silva, 2003).

Los organismos, cholgas (Aulacomya ater) y choritos (Mytilus chilensis) analizados en este estudio corresponden a muestras de bancos naturales, obtenidas por medio de muestreos mensuales, realizados entre los años 2011 a 2012, bajo el Programa de Monitoreo y Manejo de las Mareas Rojas realizado por el Servicio de Salud Magallanes de la ciudad de Punta Arenas, perteneciente a la Secretaria Regional Ministerial del Ministerio de Salud de Chile junto con el Instituto de Fomento Pesquero (IFOP).

Se seleccionaron 11 estaciones georeferenciadas entre los meses de agosto 2011 y enero 2012 considerando 4 estaciones dentro del Parque Nacional Bernardo O'Higgins (estaciones 1-4), 3 estaciones en la Reserva Nacional Alacalufes (estaciones 5, 7 y 8), 1 estación situada en el centro del Estrecho de Magallanes (estación 6) y, 3 estaciones localizadas en el interior de la Reserva de la Biósfera Cabo de Hornos (estaciones 9-11) (Tabla 1) (Fig. 1). Una vez obtenidas las muestras desde el Laboratorio de Salud de Magallanes, se trasladaron refrigeradas al Laboratorio de Química Ambiental de la Facultad de Ciencias en el Instituto de la Patagonia, Universidad de Magallanes, en donde se mantuvieron congeladas a una temperatura de $-25^{\circ} \mathrm{C}$ hasta su posterior análisis.

\section{Análisis Morfométrico y Químico Proximal}

Una vez en el laboratorio, se descongelaron las muestras y se lavaron con agua destilada para eliminar sólidos adheridos y restos de arena. A continuación, se realizó el análisis morfométrico,

Tabla 1. Estaciones, localización y periodo de muestreo seleccionados en cada lugar de estudio para la recolección de A. ater y $M$. chilensis en las distintas áreas protegidas de la región Subantártica de Magallanes.

\begin{tabular}{|c|c|c|c|c|c|}
\hline $\begin{array}{c}\text { Estaciones } \\
\qquad \mathrm{N}^{\mathrm{O}}\end{array}$ & Organismo & Localización & $\begin{array}{l}\text { Latidud } \\
\text { (S) }\end{array}$ & $\begin{array}{l}\text { Longitud } \\
\text { (W) }\end{array}$ & Área Protegida \\
\hline 1 & A. ater & Puerto Edén & $49^{\circ} 07^{\prime} 5^{\prime \prime}$ & $74^{\circ} 25^{\prime} 18^{\prime \prime}$ & $\mathrm{PNBO}^{*}$ \\
\hline 2 & A. ater & Seno Europa & $50^{\circ} 00^{\prime} 20^{\prime \prime}$ & $74^{\circ} 21^{\prime} 38^{\prime \prime}$ & $\mathrm{PNBO} *$ \\
\hline 3 & A. ater & Estero Peel & $50^{\circ} 51^{\prime} 05^{\prime \prime}$ & $74^{\circ} 05^{\prime} 54^{\prime \prime}$ & $\mathrm{PNBO} *$ \\
\hline 4 & A. ater & Estero Penguin & $49^{\circ} 55^{\prime} 11^{\prime \prime}$ & $74^{\circ} 19^{\prime} 53^{\prime \prime}$ & $\mathrm{PNBO} *$ \\
\hline 5 & M. chilensis & Estero de las Montañas & $52^{\circ} 05^{\prime} 57^{\prime \prime}$ & $73^{\circ} 17^{\prime} 6^{\prime \prime}$ & $\mathrm{RNA}^{*}$ \\
\hline 6 & M. chilensis & Bahía Gente Grande & $52^{\circ} 56^{\prime} 48^{\prime \prime}$ & $70^{\circ} 20^{\prime} 53^{\prime \prime}$ & EM \\
\hline 7 & M. chilensis & Bahía Fanny & $53^{\circ} 11^{\prime} 20^{\prime \prime}$ & $72^{\circ} 11^{\prime} 32^{\prime \prime}$ & RNA * \\
\hline 8 & M. chilensis & Cutter Cove & $53^{\circ} 22^{\prime} 9^{\prime \prime}$ & $72^{\circ} 27^{\prime} 19^{\prime \prime}$ & RNA Y y AMCPFC * \\
\hline 9 & M. chilensis & Ventisquero España & $54^{\circ} 49^{\prime} 37^{\prime \prime}$ & $69^{\circ} 46^{\prime} 17^{\prime \prime}$ & $\mathrm{RBCH}$ (Zona Núcleo) *** \\
\hline 10 & M. chilensis & Bahía Yendegaia & $54^{\circ} 52^{\prime} 57^{\prime \prime}$ & $68^{\circ} 44^{\prime} 3^{\prime \prime}$ & $\mathrm{RBCH}$ (Zona Buffer) ${ }^{* *}$ \\
\hline 11 & M. chilensis & Puerto Williams & $54^{\circ} 55^{\prime} 57^{\prime \prime}$ & $67^{\circ} 42^{\prime} 6^{\prime \prime}$ & $\mathrm{RBCH}$ (Zona Transición) ** \\
\hline
\end{tabular}

* Sielfeld (1997) / ** Rozzi et al. (2007). PNBO: Parque Nacional Bernardo O`Higgins; RNA: Reserva Nacional Alacalufes; EM: Estrecho de Magallanes; AMCRFC: Área Marina Costera Protegida Francisco Coloane y, RBCH: Reserva de la Biósfera Cabo de Hornos. 

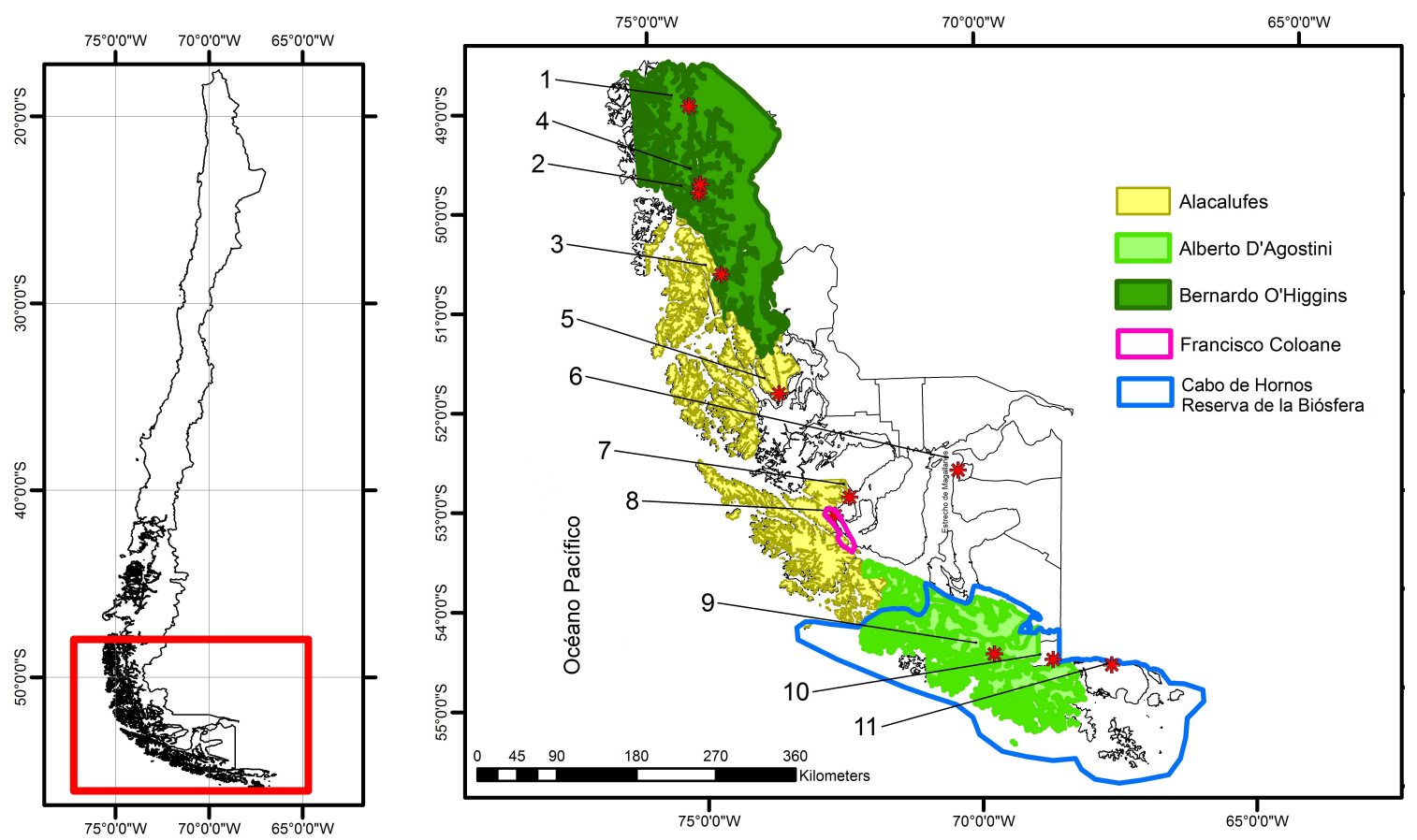

Fig. 1. Mapa de la ubicación geográfica de los sitios de muestreo, que señala las áreas protegidas de la región Subantártica de Magallanes. Estaciones del 1 al 11 se encuentran detalladas en la Tabla 1.

midiendo alto, largo y ancho de la concha, con un calibre digital $( \pm 0,01 \mathrm{~mm})$. Se extrajeron los tejidos blandos de los bivalvos, los cuales fueron pesados en una balanza analítica $( \pm 0,001 \mathrm{~g})$, para determinar el peso fresco. Posteriormente para determinar los parámetros químicos proximales, se utilizó una muestra compuesta, homogeneizada con una procesadora de alimentos, de cinco organismos de A. ater y diez de M. chilensis, de tamaño similar, con la finalidad de obtener muestras representativas de cada sector.

Los análisis de la composición química proximal se realizaron por triplicado siguiendo la metodología oficial (AOAC, 2007). La humedad se determinó por desecación en estufa hasta peso constante. Las cenizas se obtuvieron a partir de la oxidación de toda la materia orgánica por incineración y calcinación en mufla a $550^{\circ} \mathrm{C}$, hasta peso constante (NCH 1245). El método de Kjeldahl se empleó para la determinación de nitrógeno en las muestras, y se utilizó un factor de conversión de 6,25 para el cálculo de la concentración de proteínas (Astorga-España \& Mansilla, 2014).

El valor de los hidratos de carbono se estimó por diferencia. Se calculó el valor calórico de las muestras a través de la utilización de los coeficientes de Atwater (proteínas e hidratos de carbono $=4,0$ y lípidos $=9,0$ ) (Astorga-España \& Mansilla, 2014).

\section{RESULTADOS}

Los parámetros biométricos medidos para todos los organismos analizados se presentan en la Tabla 2, diferenciando según las estaciones muestreadas, y en la Tabla 3 se exhibe el promedio de los parámetros biométricos para cholgas y choritos.

Respecto de los parámetros biométricos según las estaciones de muestreo (Tabla 2 y 3), se

Tabla 3. Parámetros biométricos medios de cholgas y choritos (valor medio $\pm \mathrm{DE} ; \mathrm{n}=\mathrm{n}^{\circ}$ de organismos)

\begin{tabular}{lcc}
\hline Moluscos & $\begin{array}{c}\text { Aulacomya ater } \\
(n=85)\end{array}$ & $\begin{array}{c}\text { Mytilus chilensis } \\
(n=310)\end{array}$ \\
\hline Largo (mm) & $111,5 \pm 8,00$ & $75,7 \pm 7,64$ \\
Ancho (mm) & $55,3 \pm 5,33$ & $37,7 \pm 3,25$ \\
Alto (mm) & $37,0 \pm 4,70$ & $32,3 \pm 3,58$ \\
Peso fresco (g) & $25,3 \pm 8,50$ & $11,3 \pm 3,92$ \\
\hline
\end{tabular}


Tabla 2. Parámetros biométricos de cholgas y choritos $\left(n=\mathrm{n}^{\circ}\right.$ de organismos, promedio $\pm \mathrm{DE}$ de alto, ancho, $\mathrm{y}$ largo de la concha en $\mathrm{mm}$, y peso en $\mathrm{g}$ ), según la estación de recolección.

\begin{tabular}{ccccccc}
\hline $\begin{array}{c}\text { Estación } \\
\text { № }\end{array}$ & Organismo & $\begin{array}{c}n \\
\text { organismos }\end{array}$ & $\begin{array}{c}\text { Alto } \\
(\mathrm{mm})\end{array}$ & $\begin{array}{c}\text { Ancho } \\
(\mathrm{mm})\end{array}$ & $\begin{array}{c}\text { Largo } \\
(\mathrm{mm})\end{array}$ & $\begin{array}{c}\text { Peso } \\
(\mathrm{g})\end{array}$ \\
\hline 1 & A. ater & 30 & $39,5 \pm 14,2$ & $61,4 \pm 0,4$ & $121,9 \pm 15,8$ & $37,1 \pm 20,6$ \\
2 & A. ater & 25 & $43,0 \pm 6,1$ & $59,4 \pm 6,2$ & $118,5 \pm 10,3$ & $30,4 \pm 8,7$ \\
3 & A. ater & 5 & $30,4 \pm 2,2$ & $47,8 \pm 2,6$ & $105,9 \pm 4,5$ & $17,8 \pm 2,9$ \\
4 & A. ater & 15 & $36,9 \pm 3,4$ & $53,5 \pm 3,1$ & $105,4 \pm 7,7$ & $24,2 \pm 10,9$ \\
5 & A. ater & 10 & $35,2 \pm 1,8$ & $54,5 \pm 2,9$ & $105,9 \pm 5,8$ & $17,2 \pm 4,5$ \\
6 & M. chilensis & 50 & $37,4 \pm 3,7$ & $42,1 \pm 4,2$ & $85,9 \pm 8,9$ & $17,0 \pm 11,1$ \\
7 & M. chilensis & 50 & $31,9 \pm 2,9$ & $36,1 \pm 3,2$ & $72,7 \pm 3,9$ & $11,5 \pm 4,2$ \\
8 & M. chilensis & 60 & $26,7 \pm 3,4$ & $32,5 \pm 3,4$ & $64,2 \pm 6,6$ & $5,16 \pm 2,4$ \\
9 & M. chilensis & 50 & $34,5 \pm 3,3$ & $39,6 \pm 2,6$ & $81,9 \pm 5,3$ & $12,9 \pm 5,1$ \\
10 & M. chilensis & 50 & $30,9 \pm 3,0$ & $37,6 \pm 4,6$ & $73,1 \pm 7,6$ & $9,3 \pm 3,6$ \\
11 & M. chilensis & 50 & $32,3 \pm 4,9$ & $38,1 \pm 3,9$ & $76,2 \pm 8,7$ & $12,0 \pm 5,8$ \\
\hline
\end{tabular}

observa que las cholgas tienen los valores mayores de ancho $(61,4 \mathrm{~mm})$, largo (121,9 $\mathrm{mm})$ y cantidad de carne fresca $(37,1 \mathrm{~g})$ en la estación 1 (Puerto Edén), mientras que el ancho de la concha (43,0 $\mathrm{mm}$ ) es mas alto en la estación 2 (Seno Europa). En el caso de los choritos, todos los parámetros, alto $(37,4 \mathrm{~mm})$, ancho $(42,1 \mathrm{~mm})$, largo $(85,9 \mathrm{~mm})$ y cantidad de carne fresca $(16,96 \mathrm{~g})$, son mayores en la estación 6 (Bahía Gente Grande).

En la Tabla 4 se muestran los valores medios de la composición química obtenidos tanto para la especie A. ater, como para $M$. chilensis expresados en porcentaje de peso fresco diferenciando entre las 11 estaciones donde se recogieron las muestras.

De los resultados de la composición química o proximal (humedad, cenizas, grasas totales y proteína bruta) (Tabla 4), expresados todos en porcentaje de peso fresco, de A. ater, se observa que el valor mínimo de humedad de $74,4 \%$ corresponde a la estación 4 (Estero Penguin) y el máximo de 81,5\% en la estación 2 (Seno Europa). La cantidad mínima de grasas totales, $1,43 \%$, se presenta en la estación 2 (Seno Europa) y la máxima cantidad de grasas $1,74 \%$ en la estación

Tabla 4. Composición química expresada en g/100 g de peso fresco, diferenciando según la estación de recolección de muestra.

\begin{tabular}{cccccc}
\hline $\begin{array}{c}\text { Estación } \\
\text { № }\end{array}$ & Organismo & $\begin{array}{c}\text { Humedad } \\
(\%)\end{array}$ & $\begin{array}{c}\text { Cenizas } \\
(\%)\end{array}$ & $\begin{array}{c}\text { Grasa } \\
(\%)\end{array}$ & $\begin{array}{c}\text { Proteína } \\
(\%)\end{array}$ \\
\hline 1 & A. ater & 77,93 & 1,06 & 1,66 & 14,11 \\
2 & A. ater & 81,50 & 1,87 & 1,43 & 14,45 \\
3 & A. ater & nd & nd & nd & nd \\
4 & A. ater & 74,40 & 1,68 & 1,74 & 14,13 \\
5 & A. ater & 76,32 & 1,97 & 1,69 & 14,53 \\
6 & M. chilensis & 80,86 & 2,03 & 1,78 & 11,70 \\
7 & M. chilensis & 80,19 & 1,82 & 1,87 & 12,76 \\
8 & M. chilensis & 84,60 & 2,77 & 1,77 & 13,05 \\
9 & M. chilensis & 79,88 & 1,86 & 2,24 & 12,89 \\
10 & M. chilensis & 81,81 & 2,30 & 1,73 & 13,08 \\
11 & M. chilensis & 80,63 & 2,57 & 1,77 & 10,74 \\
\hline & & & & & nd $=$ no determinado \\
\hline
\end{tabular}


Tabla 5. Promedio y desviación estándar (mínimo-máximo) del contenido de humedad, cenizas, grasa total y proteínas expresados en $\mathrm{g} / 100 \mathrm{~g}$ de peso fresco.

\begin{tabular}{lcc}
\hline & Aulacomya ater & Mytilus chilensis \\
\hline Composición proximal & Peso fresco & Peso fresco \\
\hline Humedad (\%) & $77,5 \pm 3,01$ & $81,3 \pm 1,74$ \\
& $(74,4-81,5)$ & $(79,9-84,6)$ \\
Cenizas (\%) & $1,64 \pm 0,41$ & $2,03 \pm 0,39$ \\
& $(1,06-1,97)$ & $(1,82-2,77)$ \\
Grasa (\%) & $1,63 \pm 0,15$ & $1,87 \pm 0,19$ \\
& $(1,43-1,74)$ & $(1,73-2,24)$ \\
Proteína (\%) & $14,31 \pm 0,28$ & $12,4 \pm 0,90$ \\
& $(14,1-14,5)$ & $(10,7-13.1)$ \\
\hline Carbohidratos (\%) & 4,88 & 2,16 \\
\hline Energía (Kcal/100 g) & 91,4 & 75,2 \\
\hline
\end{tabular}

4 (Estero Penguin). En relación a las cenizas y a las proteínas, el valor más bajo se detecta en la estación 1 (Puerto Edén) con 1,06\% y 14,11\% respectivamente y el valor más alto en la estación 5 (Estero de las Montañas) que corresponde a $1,97 \%$ y $14,53 \%$ respectivamente. En cuanto a $M$. chilensis (Tabla 4), se observa que el porcentaje de humedad presenta un valor máximo de $84,60 \%$ en la estación 8 (Cutter Cove) y un valor mínimo de 79,88\% en la estación 9 (Ventisquero España), estación en la que también se determina el valor máximo de grasas totales (2,24\%, peso fresco), mientras que el valor mínimo de grasas totales se encuentra en la estación 10 (Bahía Yendegaia) $(1,73 \%)$, en la cual también se determina el valor máximo de proteínas (13,08\%). A su vez, el valor mínimo de proteínas se presenta en la estación 11 (Puerto Williams) 10,74\%.

En la Tabla 5 se presentan los valores medios, desviación estándar, así como el rango (mínimo-máximo) de la composición química obtenidos tanto para la especie A. ater, como para $M$. chilensis expresados en porcentaje de peso fresco.

Con respecto a los resultados promedios de la composición química proximal determinada en peso fresco para ambas especies del estudio (Tabla 5), se observa que la especie $M$. chilensis presenta mayores valores de humedad $(81,33 \%$,$) , cenizas$ $(2,23 \%)$ y grasas totales $(1,88 \%)$ que $A$. ater y el contenido de proteínas es menor, sin embargo, se mantienen casi constantes en todas las estaciones.
Existiendo diferencias significativas $(p<0,05)$ entre las dos especies para los contenidos de humedad y proteínas.

\section{DISCUSIÓN}

Si bien los moluscos estudiados provienen de diferentes zonas del estrecho de Magallanes, las diferencias observadas entre los choritos y las cholgas, en los parámetros biométricos, se atribuyen a las características biológicas o morfométricas propias de cada especie. Sin embargo, es de destacar que los parámetros biométricos mayores determinados en choritos son de las muestras provenientes de Bahía Gente Grande, ubicada geográficamente en el sector (5256'48”S; 70²0’53”O) (Tabla 1, Fig.1), que es el único sector de muestreo cercano a la costa de la Isla de Tierra del Fuego en el lado oriental de Estrecho de Magallanes. Probablemente, esto se deba a la ubicación que tiene la influencia de las aguas provenientes del océano Atlántico, y una relación con corrientes, mareas, tasa de sedimentación u otros factores no estudiados y correlacionados con moluscos submareales en la bibliografía (Panella et al. 1991; Antezana 1999; Ríos et al. 2010). Mientras que la mayoría de los parámetros biométricos determinados en cholgas son de las muestras provenientes de Puerto Edén ubicada geográficamente en el sector $\left(49^{\circ} 07^{\prime} 5^{\prime \prime} \mathrm{S}\right.$; 74²5'18" O) (Tabla 1, Fig. 1), en el lado occidental de Estrecho de Magallanes. Los parámetros 
biométricos determinados y la cantidad de carne medida son aspectos importantes considerados para la comercialización de este tipo de moluscos (Fuentes et al. 2009).

En los bivalvos, la composición bioquímica, proximal, de nutrientes esenciales, y la magnitud de los parámetros biométricos depende de las condiciones ambientales en que se desarrollan, y por ello presentan variaciones estacionales geográficas y con la profundidad, así como con las diferentes etapas de desarrollo (Urrutia et al. 2003; Fuentes et al. 2009). Las diferencias en la magnitud de los parámetros biométricos tienen una influencia directa en el aspecto del producto, el cual puede ser decisivo a la hora de elegir el molusco bivalvo por parte de los consumidores. Al comparar los parámetros biométricos de choritos de este estudio, con mejillones Mytilus galloprovincialis, de diferentes orígenes, elegido por ser el producto más representativo de la acuicultura española (Fuentes et al. 2009), se observa que los choritos de este estudio presentan valores mayores en todos los parámetros medidos. Esto se explica por tratarse de diferentes especies con diferentes hábitats, pero, estas comparaciones nos proveen de información sobre aspectos cualitativos de los productos magallánicos.

En este estudio, y de acuerdo a los valores de contenido proteíco y/o graso en moluscos provenientes de diferentes estaciones ubicadas en el estrecho de Magallanes, no se establece una tendencia de mayor o menor magnitud de un lugar en particular, o hacia el lado oriental u occidental del estrecho.

En general las especies de mitílidos contienen alto porcentaje de humedad en sus tejidos (Aizpun de Moreno et al. 1971; Freites et al. 2002), tal como se verifica en este estudio para los choritos con $81,3 \%$ de humedad y las cholgas con $77,5 \%$. Según datos bibliográficos (Sikorski, 1994), la proporción de humedad en los pescados blancos y en los mariscos oscila entre el 75 y $80 \%$, y este contenido de agua en los productos marinos influye directamente sobre las propiedades reológicas, el valor nutritivo y la calidad organoléptica, ejerciendo gran impacto sobre la vida comercial de estos productos.

El valor nutritivo de los productos marinos comestibles se puede determinar por diferentes parámetros, los más frecuentemente considerados son el contenido de proteínas, los niveles de aminoácidos esenciales, el contenido de grasas totales, el perfil de ácidos grasos y la concentración de minerales esenciales, etc., cuyas concentraciones son variables entre las especies (Izquierdo et al. 2000). Al comparar los valores en peso fresco de humedad, cenizas, grasas totales, proteínas, carbohidratos y energía obtenidos en este estudio, tanto para choritos, como para cholga, con valores tabulados para Mytilus edulis frescos según el INCAP (2007) donde se exponen los siguientes valores; humedad $80,58 \%$, cenizas $1,59 \%$, grasa total $2,24 \%$, proteína $11,9 \%$, carbohidratos 3,69\% y energía $86 \mathrm{Kcal} / 100 \mathrm{~g}$, se muestra que los choritos, $M$. chilensis, presentan mayor contenido de humedad, proteínas y cenizas que los $M$. edulis, y valores menores de energía, grasa total y carbohidratos. Mientras que las cholgas, presentan mayores valores de proteínas, carbohidratos y energía que los $M$. edulis y menores valores de humedad, cenizas, grasas totales.

En tanto que los valores de la Tabla de Composición de Alimentos de Chile (SchmidtHebbel et al. 1992) exhibe los siguientes valores, en peso fresco, de humedad, grasa total, proteínas y cenizas para cholgas $(81 \%, 0,8 \%, 14,2 \%$ y $1,4 \%$ respectivamente) y choritos $(82,9 \%, 1,8 \%$, $10 \%$, y $1,3 \%$ respectivamente), los cuales al ser comparados con este estudio, muestran que a excepción de la humedad, los valores de grasa, proteínas y cenizas son mayores en cholgas de este estudio. Similar caso ocurre con los choritos, en donde las grasas, cenizas y proteínas son mayores en este reporte, que las exhibidas en la tabla de composición de alimentos de Chile.

Los lípidos son uno de los componentes principales de los organismos marinos. Los mariscos coinciden con los pescados blancos en el bajo contenido graso, que se sitúa entre $0,5 \%$ y $2 \%$ en moluscos y entre 2 y $5 \%$ en crustáceos (Ávila et al. 2009). Tanto los choritos como las cholgas muestran contenidos grasos dentro de los rangos reportados en otros estudios (Ávila et al. 2009) y en las tablas de composición de alimentos INCAP (2007) Schmidt-Hebbel et al. 1992). También la cantidad de proteína bruta determinada en este estudio en choritos y cholgas, está dentro de los parámetros considerados de buena calidad y 
coincide con lo reportado por Siroski (1994), donde detalla que la cantidad de proteína bruta en pescados y mariscos oscila entre 11 y $24 \%$, dependiendo de la especie, estado nutritivo, y tipo de musculo, y que la digestibilidad de las proteínas de la carne cruda de pescado varía entre el 90 y el $98 \%$, y en moluscos y en crustáceos está alrededor del 85\%.

Las diferencias observadas en los resultados de este estudio entre choritos y cholgas, tanto en los parámetros biométricos como en los parámetros químicos, se atribuyen a las características propias de cada especie.

Los componentes nutricionales (proteína, grasa, ceniza y humedad) son importantes en la calidad de la materia prima como alimento, de acuerdo a esta afirmación los parámetros biométricos determinados y los parámetros químicos exhibidos por los moluscos $M$. chilensis y $A$. ater presentan una buena calidad nutricional, que lo hace una fuente importante para enriquecer la dieta de los habitantes de la región de Magallanes.

Estos resultados proporcionan información valiosa para el conocimiento del valor nutricional que aportan los mitílidos de poblaciones naturales de la región de Magallanes, que están sujetas a pesquerías y a la comercialización. El presente trabajo será complementado con otros parámetros específicos, de manera de contar a futuro con una mayor caracterización de los productos marinos comerciales de la zona sub-Antártica.

\section{AGRADECIMIENTOS}

Este trabajo fue financiado por por el Fondo de Innovación para la Competividad (FICRegional) Código BIP 30127771-0. Se agradece a la Seremi de Salud de Magallanes 2011, Dra. María Isabel Iduya y, al Director Zonal de Pesca en Magallanes, 2011, Sr. Ricardo Radebach por su gestión y apoyo para la obtención de muestras. A la Sra. María Teresa Fauré N., encargada del Servicio de Salud de Punta Arenas, por facilitar las muestras.

\section{LITERATURA CITADA}

Aldea, C., \& Rosenfeld, S. (2011). Macromosluscos intermareales de sustratos rocosos de la playa
Buque Quemado, Estrecho de Magallanes, sur de Chile. Revista de Biología Marina y Oceanografía, 46(2), 115-124.

Andrade, C., \& Brey, T. (2014). Trophic ecology of limpets among rocky intertidal in Bahía Laredo, estrecho de Magallanes (Chile). Anales del Instituto de la Patagonia, 42, 65-70.

Antezana, T. (1999). Hydrographic features of Magellan and Fuegian inland passages and adjacent Subantarctic waters. Scientia Marina, 63(Suppl. 1), 23-34.

Association of Official Agricultural Chemists (AOAC) (2007). Official methods of analysis of AOAC International. 18th edn. Gaithersburg: AOAC International.

Astorga-España, M. S., \& Mansilla, A. (2014). Sub-Antarctic macroalgae: opportunities for gastronomic tourism and local fisheries in the Region of Magallanes and Chilean Antarctic Territory. Journal Applied Phycology, 26, 973-978.

Astorga, M. S., Rodríguez, E. M., \& Díaz, C. (2007). Comparison of mineral and trace element concentrations in two mollusks from the Strait of Magellan (Chile). Food Composition and Analysis, 20, 273-279.

Ávila, J., Del Pozo, S., Ruiz, E., \& Varela, G. (2009). Guía nutricional del pescado, del marisco y de la conserva. Ministerio del Medio Ambiente y medio rural y marino. Disponible en: http://www.from.es/multimedia/Guianutricional_tcm85-45776.pdf

Aizpun de Moreno, J., Moreno, V. J., \& Malaspina, A. M. (1971). Estudio sobre el mejillón (Mytilus platenis d'Orb) en explotación comercial del sector bonaerense, Mar Argentino. II. Ciclo anual en los principales componentes bioquímicos (pp. 1-15). Documento Técnico FAO 21.

Cabello, A., Villarroel, R., Figueroa, B., Ramos, C., Márquez, Y., \& Vallenilla, O. (2004). Parámetros de frescura de moluscos. Revista Científica FCV-LUZ, XIV, 5, 457- 466.

Carranza, A., Defeo, O., Beck, M., \& Castilla, J. C. (2009). Linking fisheries management and conservation in bioengineering species: the case of South American mussels (Mytilidae). Reviews in Fish Biology and 
Fisheries, 19(3), 349.

Farías, A. (2008). Estado actual del cultivo y manejo de moluscos bivalvos y su proyección futura. Factores de afectan su sustentabilidad en América Latina. En A. Lovatelli, A., Farías, \& I. Uriarte (Eds,), (359 p). Puerto Montt: FAO.

FAO/WHO. (1988). Organización de las Naciones Unidas para la Agricultura y la Alimentación (FAO) - Organización Danés de Fomento Internacional (DANIDA). El pescado fresco: su calidad y cambios de calidad (pp. 1527). Roma: Colección FAO Pesca.

Fuentes, A., Fernández-Segovia, I., Escriche, I., \& Serra, J. (2009). Comparison of physicochemical parameters and composition of mussels (Mytilus galloprovincialis Lmk.) from different Spanish origins. Food Chemistry, 112, 295- 302.

Freites, L., Fernández-Reiriz, M., \& Labarta, U. (2002). Lipid classes of mussel seeds Mytilus galloprovincialis of subdital and rocky shore origin. Aquaculture, 207, 97-116.

Freites, L., Fernández-Reiriz, M., \& Labarta, U. (2003). Composición bioquímica y contenido energético del mejillón Mytilus galloprovincialis de origen submareal e intermareal: Influencia de las variables ambientales y de su origen. Ciencias Marinas, 29, 603-619.

Instituto Nacional de Capacitación Profesional (INCAP) (2007). Tabla de Composición de Alimentos de Centroamérica. M. T. Menchú \& H. Méndez, H. (Eds.), (128 pp.) (2 edn). Guatemala: INCAP/OPS.

Izquierdo, P., Torres, G., Barboza, Y., Márquez, E., \& Allara, M. (2000). Análisis proximal, perfil de ácidos grasos, aminoácidos esenciales $y$ contenido de minerales en doce especies de pescado de importancia comercial en Venezuela. Archivos Latinoamericanos de Nutrición, 50(2), 187-194.

Langley, S., Guzmán, L., \& Ríos, C. (1980). Aspectos dinámicos de Mytilus chilensis (Hupé, 1840) en el estrecho de Magallanes. I. Distribución, densidad y disposición espacial en el intermareal, Anales del Instituto de la Patagonia, 11, 319-332.
NCh1245. (Of 2001). Química de alimentos Determinación de cenizas. Instituto de salud pública. PRT-701.02-011.

Orban, E., Di Lena, G., Nevigato, T., Casini, I., Marzetti, A., \& Caproni, R. (2002). Seasonal changes in meat content, condition index and chemical composition of mussels (Mytilus galloprovincialis) cultured in two different Italian sites. Food Chemistry, 77, 57-65.

Osorio, C. (2002). Moluscos marinos en Chile, especies de importancia económica. Santiago de Chile: Facultad de Ciencias, Universidad de Chile.

Panella, S., Michelato, A., Perdicaro, R., Magazzù, G., F. Decembrini, F., \& Scarazzato, P. (1991). A preliminary contribution to understanding the hydrological characteristics of the Strait of Magellan: Austral Spring 1989. Bolletino Di Oceanologia Teorica Ed Applicata, 9, 107-126.

Ríos, C., Mutschke, E., \& Montiel, A. (2010). Estructura de la comunidad macrofaunistica bentónica en la boca oriental del estrecho de Magallanes, Chile Austral. Anales del Instituto de la Patagonia, 38, 83-96.

Rozzi, R., Massardo, F., Mansilla, A., Anderson C., Berhöfer, A., Mansilla, M., Gallardo, M., R.,... \& Barros, E. (2007). La Reserva de Biosfera Cabo de Hornos: Un desafío para la conservación de la biodiversidad e implementación del desarrollo sustentable en el extremo austral de América. Anales del Instituto de la Patagonia, 35, 55-70.

Sikorski, Z. E. (1994). Tecnología de los productos del mar: Recursos, Composición nutritiva y Conservación (pp. 39-70). Madrid: Editorial Acribia.

Sielfeld, W. (1997). Las áreas Protegidas de la XII Región de Chile en la Perspectiva de los Mamíferos Marinos. Estudios Oceanológicos, 16, 87-107.

Schmidt-Hebbel, H., Pennacchiotti, I., Masson, L., \& Mella, M. (1992). Tabla de composición química de alimentos chilenos. Santiago de Chile: Universidad de Chile.

Uriarte, I. (2008). Estado actual del cultivo y manejo de moluscos bivalvos y so proyección futura. Factores de afectan su sustentabilidad en 
América Latina. Lovatelli, A., Farías, A., \& Uriarte, I. (Eds,) (359 pp.). Puerto Montt: FAO.

Valdenegro, A., \& Silva. N. (2003). Caracterización oceanográfica física y química de la zona de canales y fiordos australes de Chile entre el estrecho de Magallanes y cabo de Hornos
(CIMAR 3 Fiordos). Ciencia y Tecnología del Mar, 26(2), 19-60.

Zuñiga, O. (2002). Guía de Biodiversidad. No 1. Vol. 1: Macrofauna y Algas Marinas: Moluscos. CREA: Centro regional de estudios y Educación Ambiental, II Región de Antofagasta. Chile. 